\title{
Sliding-Mode Control of Nonlinear Discrete-Input Pneumatic Actuators
}

\author{
Sean Hodgson ${ }^{1}$, Minh Quyen Le ${ }^{2}$, Mahdi Tavakoli ${ }^{1}$, Minh Tu Pham ${ }^{2}$ \\ ${ }^{1}$ Department of Electrical and Computer Engineering, University of Alberta, Edmonton, AB Canada T6G2V4 \\ ${ }^{2}$ Laboratoire Ampère, UMR CNRS 5005, Université de Lyon, INSA-LYON, F-69621 Villeurbanne Cedex, France
}

\begin{abstract}
This paper proposes a sliding mode law for precise position control with minimal switching activity for a robotic system that uses on/off (solenoid) pneumatic actuators, For a two-chamber pneumatic actuator with four binary solenoid valves, there is a total of sixteen possible input combinations defined directly from the state of the four on/off solenoid valves present in the system. However, only seven of these discrete operating modes are considered both functional and unique. Accordingly, we use a seven-mode sliding controller that minimizes the position error using modes that have both the necessary and sufficient amounts of drive energy and, thus, involve reduced switching activity. An analysis of the closedloop system stability is carried out. The performance of the proposed control design is experimentally verified on a single pneumatic actuator comprising of two chambers driven by four on/off solenoid valves.
\end{abstract}

Index Terms-Pneumatic actuator, on/off solenoid valve, robot control, sliding mode control, position tracking, switching activity, stability.

\section{INTRODUCTION}

Recent developments in robotics and telerobotics have allowed working in environments that are normally difficult or hazardous for the human hand to reach or operate in. Robotic systems have been developed for applications ranging from surgery to space exploration. In robot-assisted surgery, for instance, robots may be required to work in areas that have strong magnetic fields. Magnetic Resonance Imaging (MRI) affords images with high resolution and contrast and allows surgeons to access a patient's three-dimensional visualization in real-time [1].

Pneumatic actuators offer many advantages for positioning applications in addition to being inert to magnetic fields: low maintenance cost, high ratio of power to weight, cleanliness and safety are some of these advantages [2]. However, they suffer from common drawbacks including friction and sensitivity of actuator dynamics to load and piston position along the cylinder stroke [3]. Also, from a control perspective, controlling a pneumatic actuator is a challenge because the system dynamics are highly non-linear [4].

The non-linear nature of a pneumatics actuator is exacerbated when it uses on/off solenoid valves, which have wide-spread applications due to the high cost of servovalves (due to the precision machining required in their manufacturing) [5]. In this case, precise control is difficult due to the discrete-input nature of the system. Most solenoidvalve pneumatic systems utilize a pulse width modulated (PWM) controller. Using time averaging, a PWM input with a sufficiently high frequency can approximate the continuous input properties of a servo-valve [6]. Regardless of whether

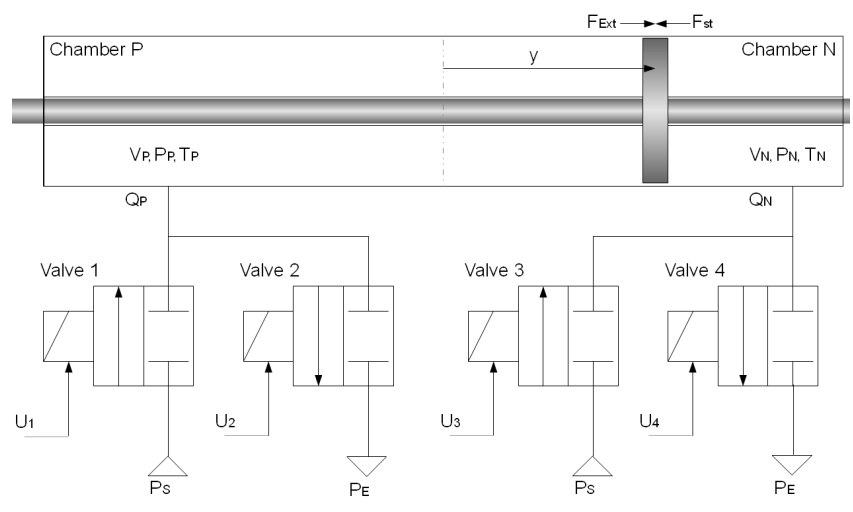

Fig. 1. Electro-pneumatic system with 4 on/off solenoid valves

a servo-valve or PWM input is utilized, a control method must be chosen to compensate for the non-linearities of the system. In [2] the nonlinear system is transformed into an equivalent linear system (controlled by PWM). Because of the highly nonlinear nature of a pneumatic system it is better to address its control with a nonlinear model based control. Other research areas use non-linear averaging techniques based on a sliding mode approach to control pneumatic systems [3], [4], [6], [7].

Sliding mode control is a form of variable structure control that alters the dynamics of a nonlinear system by the application of a high-frequency switching control [8]. Sliding mode control can account for the non-linearities of the system as well as its dynamic uncertainties [5]. In this paper we consider a pneumatic actuator comprised of two chambers as shown in Figure 1. Each chamber has two solenoid valves, each of which can be either locked or connected to a compressed air supply (source pressure) or to exhaust (atmosphere pressure). Since we cannot have a chamber connected to both pressure and exhaust at the same time, only nine discrete modes exist. We assume the three modes where both chambers are locked, venting, and pressurizing are functionally equivalent, then we can assume the system has a total of seven unique discrete modes.

The sliding control system utilizes discrete operating modes defined directly from the state of the on/off solenoid valves, with the switching between these discrete modes decided based on the current tracking error, thereby bypassing the need for a PWM input. In [5], three discrete modes are considered for a two-chamber actuator (see Figure 1): two modes defined by connecting one chamber to exhaust and connecting one chamber to pressure (and the reverse thereof) 
and one mode defined by closing all valves.

In this paper, we expand the three-mode open-loop model of [5] into a seven-mode open-loop model. The new system has four extra modes defined by opening only one of the four solenoid valves at a time, which facilitate appropriate amounts of drive energy for good positioning precision and reduced switching activity.

Limiting the drive power allows us to make smaller and fewer adjustments, improving the positioning accuracy of the controlled system. Compared to the 3 mode paper we are extending to 7 modes and providing guidelines for the parameters $\tau, \beta$ and $\epsilon$, which will be discussed later.

We will decide the thresholds for transition from one control mode to another control mode based on the full state of the system: piston position, velocity, and acceleration as well as chamber pressures. By optimizing these transition thresholds, we can decrease the open-close activity of the on/off solenoid valves, which increases their operating lifespan.

The organization of this paper is as follows: The modeling of the pneumatic actuator with its chamber and 4 solenoid valves is reported in Section II. The discrete input model of the actuator is given in Section III. The design and theoretical analysis of the sliding controller are discussed in Section IV. The simulation study to verify the control laws are presented in this paper in Section $\mathrm{V}$ The experimental results are presented in this paper in Section VI. Finally, the concluding remarks are presented in Section VII.

\section{Modeling of the Open-Loop Pneumatic ACTUATOR}

This section of the paper derives the open-loop model of the pneumatic actuator including its on/off solenoid valves as shown in Figure 1. To describe the air flow dynamics in a cylinder, we assume that: Air is a perfect gas and its kinetic energy is negligible in the chamber, the pressure and the temperature are homogeneous in each chamber, the evolution of the gas in each chamber is polytropic, with the exception of some controller thresholds which were selected using isothermic assumptions. The temperature variation in chambers is negligible with respect to the supply temperature. The mass flow rate leakages are negligible, and the supply and exhaust pressures are constant.

The Simscape simulations in Section V does not assume/model all of these assumptions.

\section{A. Model of the Pneumatic Chambers}

If the charging and discharging of the chambers' cylinders are assumed to be polytropic, then the pressure dynamics of these chambers can be approximated [9] as

$\dot{P}_{P}=\frac{k}{V_{P}}\left(r T Q_{P}-A P_{P} \dot{y}\right) \quad \dot{P}_{N}=\frac{k}{V_{N}}\left(r T Q_{N}+A P_{N} \dot{y}\right)$

where $P_{P}$ and $P_{N}$ refer to pressures (Pa) inside the chambers $\mathrm{P}$ and $\mathrm{N}$, respectively, $V_{P}$ and $V_{N}$ refer to volumes $\left(\mathrm{m}^{3}\right)$ of the chambers $\mathrm{P}$ and $\mathrm{N}$, respectively, $Q_{P}$ and $Q_{N}$ refer to mass flow rates $(\mathrm{kg} / \mathrm{s})$ of the chambers $\mathrm{P}$ and $\mathrm{N}$, respectively, $A$ refers to the piston cylinder area $\left(\mathrm{m}^{2}\right), T$ refers to the chamber temperature $(\mathrm{K}), k$ refers to the polytropic constant, $r$ refers to the universal gas constant $(\mathrm{J} /(\mathrm{kgK}))$, and $y$ refers to the piston position $(\mathrm{m})$ shown in Figure 1. Note the arrows for position $y$, Force $(\mathrm{N}) F_{E x t}$ and $F_{s t}$ shown in Figure 1 refer to their positive directions, a negative magnitude to any of these quantities will refer to the opposite direction.

\section{B. Model of the valves}

The mass flow rates $Q_{P}$ and $Q_{N}$ can be derived in terms of the discrete voltage inputs $U_{1}, U_{2}, U_{3}$ and $U_{4}$ shown in Figure 1 and the continuous pressure inputs $P_{P}$ and $P_{N}$ :

$$
\begin{aligned}
& Q_{P}=U_{1} Q\left(P_{S}, P_{P}\right)-U_{2} Q\left(P_{P}, P_{E}\right) \\
& Q_{N}=U_{3} Q\left(P_{S}, P_{N}\right)-U_{4} Q\left(P_{N}, P_{E}\right)
\end{aligned}
$$

Here, $P_{S}$ and $P_{E}$ are the pressures of the supply and the exhaust. In general, $\mathrm{Q}\left(P_{U p}, P_{\text {Down }}\right)$ used in $(2 \mathrm{a})$ and $(2 \mathrm{~b})$, in which $P_{U p}$ is the upstream pressure and $P_{\text {Down }}$ is the downstream pressure, refers to the expression for the mass flow rate through an orifice. This generalized model has two parameters to describe the mass flow rate: the critical pressure ratio $b=0.433$ and the sonic conductance $C_{v a l}$ (mass flow rate constant) [10]:

$$
\begin{aligned}
& Q\left(P_{U p}, P_{\text {Down }}\right)=C_{v a l} P_{U p} \sqrt{\frac{T_{\text {Atm }}}{T_{U p}}} \times \\
& \begin{cases}\sqrt{1-\left(\frac{\frac{P_{\text {Down }}}{P_{U p}}-b}{1-b}\right)^{2}} & \text {,if } \frac{P_{\text {Down }}}{P_{U p}}>b \text { (subsonic) } \\
1 & \text {,if } \frac{P_{\text {Down }}}{P_{U p}} \leq b \text { (choked) }\end{cases}
\end{aligned}
$$

In the above, $T_{U p}$ is the upstream temperature of air and $T_{A t m}$ is the atmospheric temperature. Also, $C_{V a l}$ is a characteristic of the valve.

\section{Model of the Piston}

Finally, the dynamics of the mechanical actuator involving the applied force on the piston and the resulting piston motion is

$$
A\left(P_{P}-P_{N}\right)-b_{m} \dot{y}+F_{E x t}-F_{S t}=M_{m} \ddot{y}
$$

where $b_{m}$ is the viscose coefficient $\left.(\mathrm{N} \mathrm{s} / \mathrm{m})\right), M_{m}$ is the total mass of the load and the piston $(\mathrm{Kg}), F_{S t}$ is the stiction force $(\mathrm{N})$, and $F_{E x t}$ is the external force $(\mathrm{N})$. For simplicity, the stiction force and external force is assumed to be negligible. The $F_{S t}$ was considered to be negligible since the pneumatic actuator used in experiment was an Airpel anti-stiction cylinder (ALL AIR Inc, New York, US).

\section{Discrete INPUT MODEL OF THE OPEN-LOOP ACTUATOR}

It is possible to combine the equations in Section II to write the dynamics of the open-loop pneumatic actuator in a 7-mode discrete-input. Differentiating (4) and substituting 
(1) in it, the dynamics of the actuator are obtained as

$$
\dddot{y}=F(Z)+\frac{k r T}{M_{m}}\left(\frac{Q_{P}}{l / 2+y}-\frac{Q_{N}}{l / 2-y}\right)
$$

where $Z=\left\{y, \dot{y}, \ddot{y}, P_{P}, P_{N}\right\}$ is the state vector and

$$
F(Z)=\frac{-b_{m}}{M_{m}} \ddot{y}-\frac{A k}{M_{m}}\left(\frac{P_{P}}{l / 2+y}+\frac{P_{N}}{l / 2-y}\right) \dot{y}
$$

where $l$ is the total length of the chamber. We find that there are a total of nine discrete modes for the solenoid valves [9]. These modes are shown in Table I. This paper will outline a controller that only utilizes modes $M_{1}$ through $M_{7}$ of the open-loop system. For the seven discrete modes, dynamic

TABLE I

Nine Discrete Modes of The Open-Loop ACtuAtor

\begin{tabular}{cccccccc|cc}
\hline \hline & $M_{1}$ & $M_{2}$ & $M_{3}$ & $M_{4}$ & $M_{5}$ & $M_{6}$ & $M_{7}$ & $M_{8}$ & $M_{9}$ \\
\hline$U_{1}$ & 0 & 1 & 0 & 0 & 0 & 1 & 0 & 0 & 1 \\
\hline$U_{2}$ & 0 & 0 & 1 & 0 & 0 & 0 & 1 & 1 & 0 \\
\hline$U_{3}$ & 0 & 0 & 0 & 0 & 1 & 0 & 1 & 0 & 1 \\
\hline$U_{4}$ & 0 & 0 & 0 & 1 & 0 & 1 & 0 & 1 & 0 \\
\hline \hline
\end{tabular}

equation can be obtained by substituting (2a) and (2b) into (5). We obtain

$$
\begin{aligned}
& \dddot{y}= \begin{cases}F(Z) & , \text { mode } M_{1} \\
F(Z)+(-1)^{i} B_{i}(Z) & , \text { mode } M_{i} \neq M_{1}\end{cases} \\
& B_{2}(Z)=\frac{k r T}{M_{m}} \frac{Q\left(P_{S}, P_{P}\right)}{(l / 2+y)} \quad B_{3}(Z)=\frac{k r T}{M_{m}} \frac{Q\left(P_{P}, P_{E}\right)}{(l / 2+y)} \\
& B_{4}(Z)=\frac{k r T}{M_{m}} \frac{Q\left(P_{N}, P_{E}\right)}{(l / 2-y)} \quad B_{5}(Z)=\frac{k r T}{M_{m}} \frac{Q\left(P_{S}, P_{N}\right)}{(l / 2-y)} \\
& B_{6}(Z)=B_{2}(Z)+B_{4}(Z) \quad B_{7}(Z)=B_{5}(Z)+B_{3}(Z)
\end{aligned}
$$

Note that because $P_{E} \leq P_{P} \leq P_{S}, P_{E} \leq P_{N} \leq P_{S}$ and $-l / 2 \leq y \leq l / 2$ and mass flow rates are non-negative functions $B_{2}(Z)$ through $B_{7}(Z)$ are all positive or equal to zero. The value $i$ takes a range of integer values from 2 to 7.

\section{The Sliding Mode Controller Design}

For a position-controlled system, we can define the following sliding surface $s=0$ where $s$ is defined as:

$$
s=\frac{\ddot{e}}{\omega^{2}}+\frac{2 \xi \dot{e}}{\omega}+e
$$

where $e$ is the position error $y-y_{d}, y$ is the actual position, $y_{d}$ is the desired position, and $\xi$ and $\omega$ are constant and positive numbers. We use this function $s$ and invoke the seven different modes of the open-loop system based on five different regions of the function $s$. These regions of $s$ and the selected operating mode of the system are illustrated in Table II. Please note that the input voltages (i.e., control actions) for each mode is listed in Table I.
TABLE II

SELECTION OF THE OPERATING MODE BASED ON POSITIONING ERROR $s$

\begin{tabular}{ccc}
\hline \hline Region & $\begin{array}{c}\text { Discrete } \\
\text { operating modes }\end{array}$ & $\begin{array}{c}\text { Magnitude of } \dot{s} \\
\text { from (10) }\end{array}$ \\
\hline$s>\beta$ & $M_{7}$ & Large negative \\
$\beta \geq s>\epsilon$ & $M_{3}$ and $M_{5}$ & Modest negative \\
$\epsilon \geq s>-\epsilon$ & $M_{1}$ & Minimal \\
$-\epsilon \geq s>-\beta$ & $M_{2}$ and $M_{4}$ & Modest positive \\
$-\beta \geq s$ & $M_{6}$ & Large positive \\
\hline \hline
\end{tabular}

\section{A. Stability}

To be able to analyze stability, consider the Lyapunov function candidate

$$
V=\frac{1}{2} s^{2}
$$

$V$ is a positive-valued function, therefore if $\dot{V}<0, V$ and $|s|$ will be decreasing. Assuming $s$ is initially bounded, $s$ will asymptotically approach zero if we control the system so that

$$
\dot{V}=\dot{s} s<-\eta|s|
$$

for some constant $\eta>0$ [8][5]. Take the derivative of (7) and substitute (6) to obtain

$$
\dot{s}= \begin{cases}\lambda & , \text { mode } M_{1} \\ \lambda+(-1)^{i} B_{i}(Z) / \omega^{2} & , \text { mode } M_{i} \neq M_{1}\end{cases}
$$

where $\lambda=\left(F(Z)-\dddot{y}_{d}\right) / \omega^{2}+2 \xi \ddot{e} / \omega+\dot{e} . \lambda$ will be bounded if $\dot{y}, \ddot{y}, \dot{y}_{d}, \ddot{y}_{d}, \dddot{y}_{d}, P_{P}$ and $P_{N}$ are bounded. $P_{P}$ and $P_{N}$ are bounded between $P_{S}$ and $P_{E} . y_{d}$ is a controlled input to the system thus $y_{d}, \dot{y}_{d}, \ddot{y}_{d}, \dddot{y}_{d}$ are assumed to be bounded. If we rewrite the (4) as

$$
M_{m} \ddot{y}+b_{m} \dot{y}=A\left(P_{P}-P_{N}\right)
$$

we see that the right side of (11) is always bounded. Therefore velocity is a $1^{\text {st }}$ order differential equation, which means velocity is a decaying exponential. Therefore if $\dot{y}$ is initially bounded, then $\dot{y}$ will always be bounded. If we rewrite (4)

$$
\ddot{y}=\frac{1}{M_{m}}\left(A\left(P_{P}-P_{N}\right)-b_{m} \dot{y}\right)
$$

We find that it is defined purely in terms of bounded functions, thus it too must always be bounded. Therefore $\lambda$ is also bounded.

Therefore if the positive-valued functions $B_{i}(Z)$ are sufficiently large then modes $M_{2}, M_{4}$ and $M_{6}$ can ensure $\dot{s}>\eta$ and the modes $M_{3}, M_{5}$ and $M_{7}$ can ensure $\dot{s}<-\eta$.

All six functions $B_{i}(Z)$ are linearly proportional to $C_{v a l}$ and $P_{S}$, the valve's mass flow rate constant in (3), thus choosing a large enough valve or supply pressure will ensure that these scalar functions will be sufficient in magnitude. Thus, using the modes $M_{2}, M_{4}$ and $M_{6}$ when $s<0$, and using $M_{3}, M_{5}$ and $M_{7}$ when $s>0$. Will ensure (9), and thus the convergence of $s$ to zero over time.

if $s$ converges to zero and $y_{d}, \dot{y}_{d}, \ddot{y}_{d}$ are bounded the 
output $y, \dot{y}, \ddot{y}$ will also be bounded and thus the system will be BIBO stable.

\section{B. Controller Mode Selection}

The 7-mode controller requires knowledge of the current chamber pressures to pick the appropriate operating modes. This requires additional sensors as compared to the 3-mode controller. These sensors are required for deciding which control mode to use when $\beta \geq|s|>\epsilon$ (see Table II).

We will first study the negative region $-\beta \geq s>-\epsilon$, if we evaluate (4) under mode $M_{2}$ and assume that the filling chamber has a sufficient amount of time to be fully pressurized we can see that the $\ddot{y} \propto\left(P_{S}-P_{N}\right)$. If we evaluate (4) under mode $M_{4}$ and assume that the venting chamber has a sufficient amount of time to be fully vented we can see that the $\ddot{y} \propto\left(P_{P}-P_{E}\right)$ If we define

$$
\begin{aligned}
E_{1} & =\left(P_{S}-P_{N}\right)-\left(P_{P}-P_{E}\right) \\
& =\left(P_{S}+P_{E}\right)-\left(P_{P}+P_{N}\right)
\end{aligned}
$$

the magnitude of $E_{1}$ is positive when the pressure difference $P_{S}-P_{N}$ is greater than the pressure difference $P_{P}-P_{E}$. Therefore, when $E_{1}$ is positive, the appropriate operating mode for the region $-\epsilon \geq s>-\beta$ is $M_{2}$ as it will result in a higher piston acceleration compared to the mode $M_{4}$. Conversely when the magnitude of $E_{1}$ is negative $M_{4}$ will result in a higher piston acceleration compared to $M_{2}$. In the positive region $\beta \geq s>\epsilon$, comparing modes $M_{5}$ and $M_{3}$, from (4) we see that the magnitudes of $\ddot{y}$ are based on the pressure differences $P_{S}-P_{P}$ and $P_{N}-P_{E}$ respectively. Let us define

$$
E_{2}=\left(P_{S}-P_{P}\right)-\left(P_{N}-P_{E}\right)=E_{1}
$$

The magnitude of $E_{2}$ is positive when the pressure difference $P_{S}-P_{P}$ is greater then the pressure difference $P_{N}-P_{E}$. Therefore when $E_{2}$ is positive the appropriate operating mode for the region $\epsilon \geq s>\beta$ is $M_{5}$ as it will accelerate the piston more compared to mode $M_{3}$. Conversely when the magnitude of $E_{2}$ is negative mode $M_{3}$ will result in a higher piston acceleration compared to $M_{5}$ In summary, the magnitude of $s$ in (7) and the magnitude of $E_{1}$ in (13) can be used by the controller to select the best of the seven operating modes see Figure 2.

\section{Selecting Parameters $\tau, \beta$ and $\epsilon$}

This section proposes appropriate ways for selecting the controller parameters of $\tau, \beta$ and $\epsilon$ for smoothest motions and least switching activity.

1) Selecting $\tau$ : The purpose of the timeout parameter $\tau$ is to reduce switching between the modes used in the region $\beta \geq|s|>\epsilon$ by enforcing a minimum amount of time between modes transitions in this region. A larger $\tau$ value will reduce switching. A smaller $\tau$ value will give a more accurate output. There is a trade-off between these goals, therefore an appropriate value of $\tau$ should be determined by evaluating the open-loop responses $M_{2}$ and $M_{4}$. For initial conditions (at $t=0$ ) of $y=0$ and $V_{P}=V_{N}=A \frac{l}{2}$ (i.e., the piston positioned in halfway along the cylinder length), using

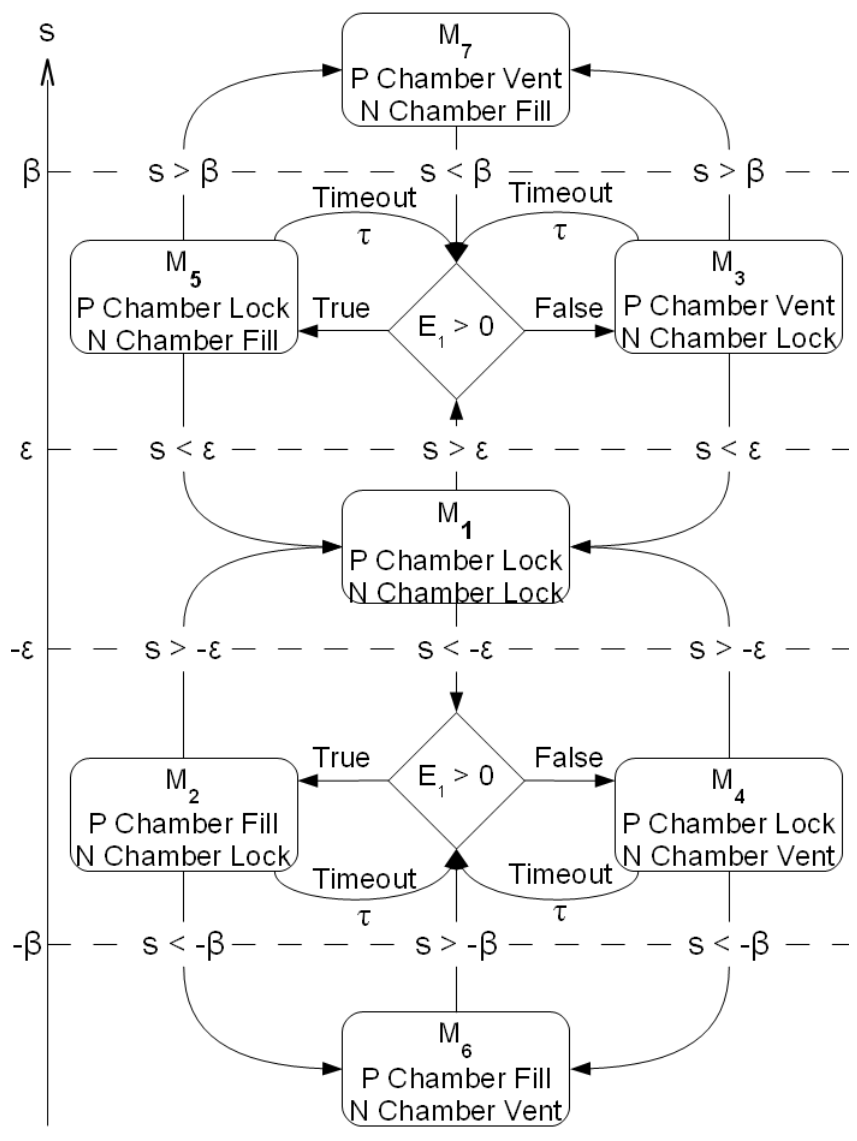

Fig. 2. 7-mode controller diagram

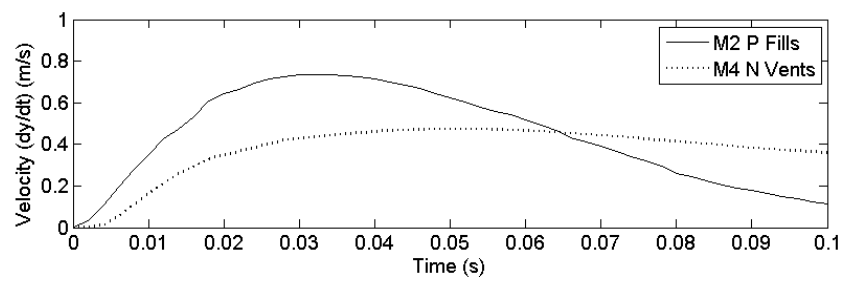

Fig. 3. Open-loop responses modes $M_{2}$ and $M_{4}$

(1) - (4) we can simulate the open-loop system response in each of these modes (Figure 3). These initial conditions where selected because they are symmetric in nature.

For the pressurizing case $M_{2}$ the intial conditions $P_{P}=$ $P_{N}=P_{E}$ was selected. For the venting case $M_{4}$ the intial conditions $P_{P}=P_{N}=P_{S}$ was selected.

We define $t_{\text {fill }}$ as the time it takes to reach the maximum velocity $\max (\dot{y})$ for the $M_{2}$ Chamber $\mathrm{P}$ filling profile and $t_{v e n t}$ as the time it takes to reach the maximum velocity $\max (\dot{y})$ for the $M_{4}$ Chamber $\mathrm{N}$ venting profile. If we compare $t_{\text {fill }}$ and $t_{\text {vent }}$ obtained from simulation we see that $t_{f i l l}$ is a shorter span of time. For our system tests we have selected $\tau=t_{\text {fill }}$.

2) Selecting $\beta$ : The magnitude $\beta$ is the transition threshold between a mode connecting the two chambers to supply and exhaust pressures (i.e., $M_{6}$ or $M_{7}$ ), and the alternating modes for opening only a single valve (i.e., $M_{2} / M_{4}$ or 
$\left.M_{3} / M_{5}\right)$.

For our purposes $\beta$ was chosen empirically. We used the following equation as a starting point.

$$
\beta=\frac{3 A\left(P_{S}-P_{E}\right)}{8 \omega b_{m}}
$$

A higher $\beta$ was found to be more optimal for $y_{r e f}$ signals with large variation and a smaller $\beta$ was found to be more optimal for $y_{r e f}$ signals with smaller variation.

For our experiments the we selected a $\beta=3 \mathrm{~mm}$. This selection of $\beta$ is not unique however it has been demonstrated in our results as being appropriate.

3) Selecting $\epsilon$ : When the magnitude of $s$ is less than a positive valued but small $\epsilon$, the controller removes the actuation from the system by closing all valves. The magnitude of $\epsilon$ should ideally be selected so that when this occurs and the effect of past actuations settles out, the position difference, $y-y_{d}$, will be less than some desired small amount $e_{\min }$. Therefore, $\epsilon$ is selected as $\epsilon=e_{\min }$

\section{Simulation Study}

To analyze the performance of the 7-mode controller described in this paper we will be comparing it against the original 3-mode controller it was based on in [5].

\section{A. Simulation Parameters and Test Inputs}

For our simulation, we selected the model parameters listed in Table III. These model parameters correspond to the experimental setup that will be used in Section VI. The

TABLE III

System Parameters TABLE

\begin{tabular}{|c|c|c|}
\hline Var. & Value & Label \\
\hline$l$ & $0.1 \mathrm{~m}$ & Chamber Length \\
\hline$T$ & $23 \mathrm{C}$ & Chamber Temperature \\
\hline$C_{v a l}$ & $3.4 \times 10^{-9} \mathrm{~kg} /(\mathrm{s} \mathrm{Pa})$ & Mass Flow Rate Const. \\
\hline$P_{S}$ & $300,000 \mathrm{~Pa}$ & Supply Air Pressure \\
\hline$P_{E}$ & $100,000 \mathrm{~Pa}$ & Exhaust Air Pressure \\
\hline$k$ & 1.2 & polytropic constant \\
\hline$A$ & $1.814 \mathrm{~cm}^{2}$ & Piston Cylinder Area \\
\hline$b_{m}$ & $50(\mathrm{~N} \mathrm{~s}) / \mathrm{m}$ & Viscosity Coefficient \\
\hline$M_{m}$ & $0.9 \mathrm{~kg}$ & Total Mass of load \\
\hline
\end{tabular}

following controller parameters were selected: $\omega=100 \mathrm{rad} / \mathrm{s}$, $\xi=0.5, \tau=40 \mathrm{~ms}, \beta=3 \mathrm{~mm}$ and $\epsilon=1 \mathrm{~mm}$. To model our system we utilized the Simulink Simscape toolbox.

\section{B. Simulation Results}

The simulation was run utilizing a $40 \mathrm{~mm}$ peak-peak sine wave test input with frequencies varying from $0.1 \mathrm{~Hz}-3.0 \mathrm{~Hz}$, the results of the simulations are charted in Figure 4. From these results, we find that for both the 3-mode and the 7mode systems increasing the input frequency leads to an increasing RMS tracking error

In the 7 mode system, for moderate differences between $y_{d}$ and $y$, this difference may not be enough to switch from the $|s|>\beta$ region. As we can see this can cause an

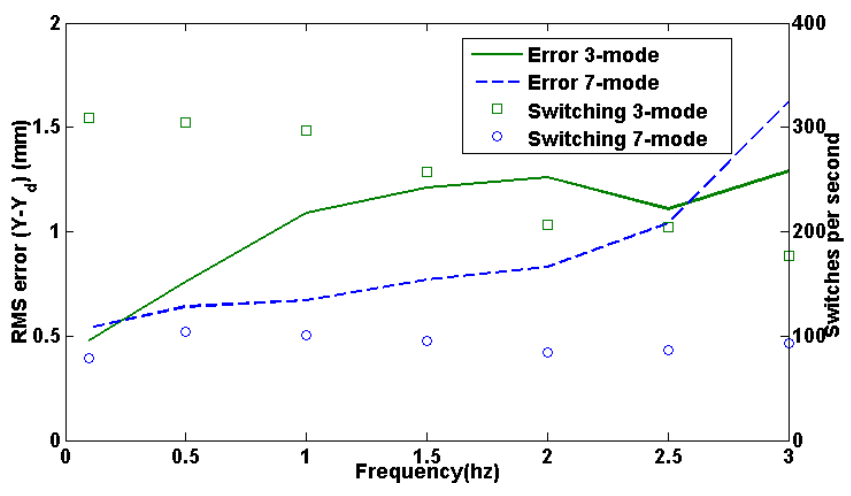

Fig. 4. Sine-wave simulation results

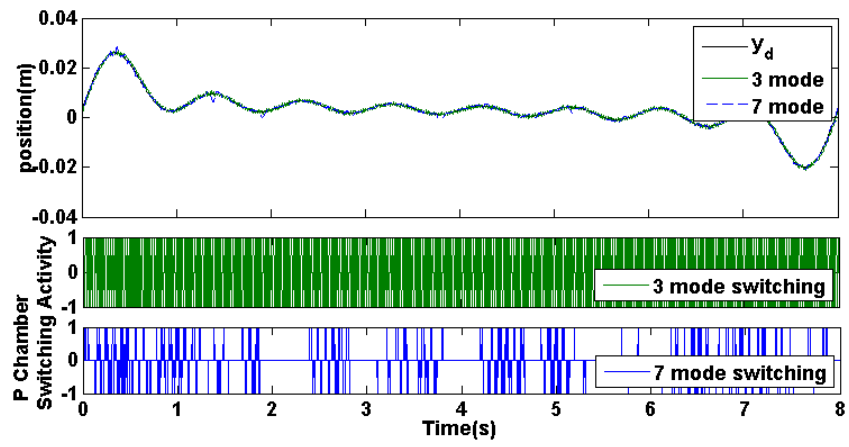

Fig. 5. Multiple summed sine-wave simulation results

increase in tracking error in the $2-3 \mathrm{hz}$ range for the 7 -mode system. This is one of the costs of using the 7-mode sliding control algorithm. The advantage of the 7-mode controller algorithm is the reduction in the solenoid valves switching as observed in Figure 4. At $1.5 \mathrm{~Hz}$, this is equal to $63 \%$ reduction in switching activity and $0.45 \mathrm{~mm}$ improvement in tracking error.

To test the system with a more complicated signal, we used an input that is a summation of 8 sine waves with 8 different frequencies. As we can see from Figure 5, both controllers have no trouble tracking the multi-sine wave; however the switching activity for the 7-mode controller is greatly reduced compared to the 3-mode controller. From the 3 -mode controller to the 7 -mode controller, there is a $64 \%$ reduction in solenoid switching activity.

\section{EXPERIMENTAL TESTING}

In this section, experiments with a 1-DOF system are reported. This experimental setup is the same as the one described in [11] for a single actuator. For this experiment, the following controller parameters were selected were the same as in simulation except $\omega=60 \mathrm{rad} / \mathrm{s}$.

\section{A. Experimental Results}

The experiment was run utilizing a $40 \mathrm{~mm}$ peak-peak sine wave test input with frequencies varying from $0.1 \mathrm{~Hz}-3.0 \mathrm{~Hz}$. The recorded results from these experiments are charted in Figure 6. When we compare these results for the 3-mode controller and the 7-mode controller, we can see that there 


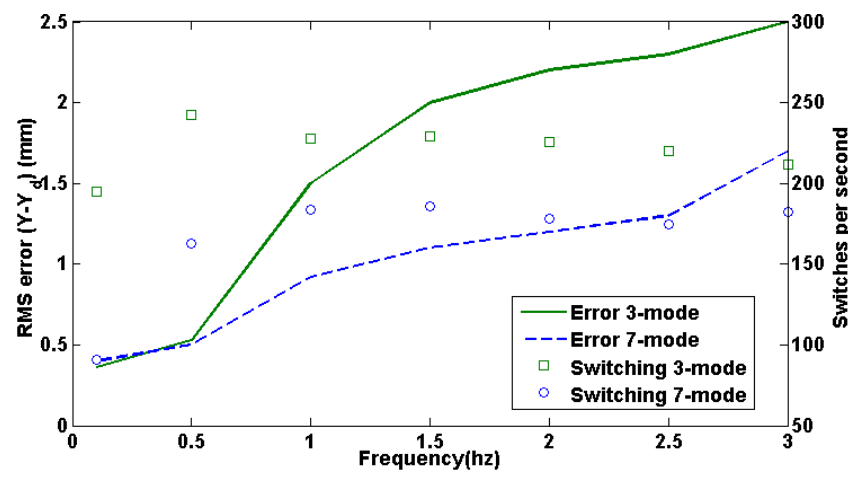

Fig. 6. Sine-wave experimental results

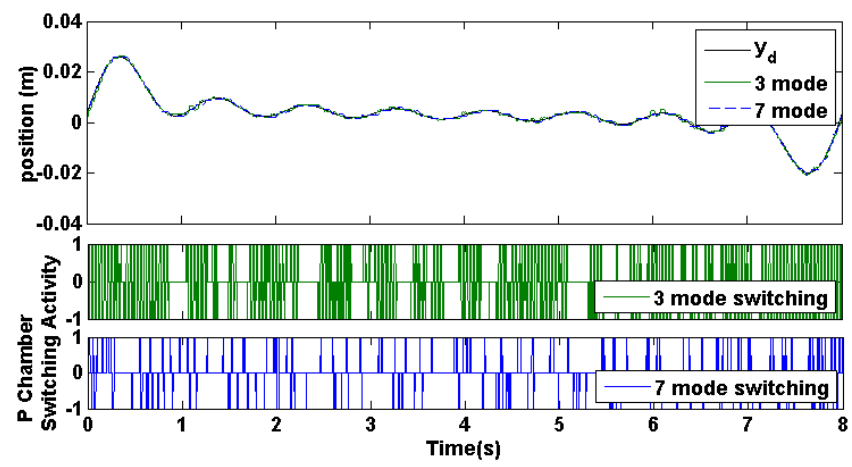

Fig. 7. Multiple summed sine-wave experimental results

was a notable improvement to tracking performance and switching activity for the 7-mode controller (compared to the 3-mode controller).

To test the system with a more complicated signal, we used an input that is a summation of 8 sine waves with 8 different frequencies (exact same as in simulation). As we can see from Figure 5, both controllers have no trouble tracking the multi-sine wave; however the switching activity for the 7-mode controller is reduced compared to the 3mode controller. From the 3-mode controller to the 7-mode controller, there is a $49 \%$ reduction in solenoid switching activity.

To test the load disturbance rejection of the algorithm a $1 \mathrm{~kg}$ weight was attached to the actuator using a rope and a pulley. The experiment was run utilizing the sine wave test input with a $1.5 \mathrm{~Hz}$ frequency. The recorded results are shown in Figure 8. Tracking error for both 3 mode and 7 mode increased by $0.2 \mathrm{~mm}$ RMS. Switching was found to increase for both 3 mode and 7 mode by $\sim 10 \%$.

\section{CONCLUDING REMARKS}

This paper proposed a sliding mode law for precise position control with minimal switching activity designed for use on a pneumatic actuator. The two-chamber actuator with four on/off solenoid valves had a total of sixteen possible modes only seven of which were considered functional and unique and were used for this particular control law. This controller uses four more additional modes compared to [5] that decrease the coarseness in the drive force for lower

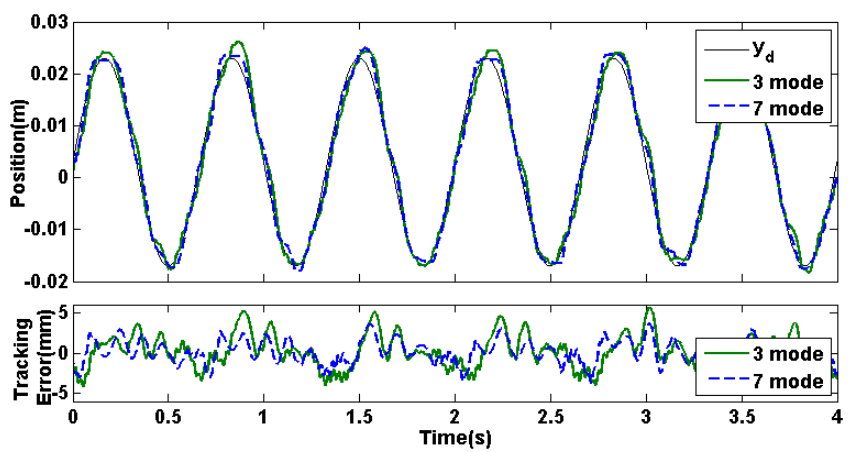

Fig. 8. Sine Wave $1.5 \mathrm{~Hz}$ with $1 \mathrm{~kg}$ load attached to actuator

position tracking errors. The main advantage of the 7-mode controller is a reduction in the switching of the solenoid valves compared to the 3-mode control. The tracking error performance was experimentally found to improve in 7-mode as compared to 3-mode control.

\section{REFERENCES}

[1] M. Oura, Y. Kobayashi, J. Okamoto, and M. Fujie, "Development of MRI compatible versatile manipulator for minimally invasive surgery," in Proceedings of 2006 First IEEE/RAS-EMBS International Conference on Biomedical Robotics and Biomechatronics (BIOROB), Tuscany, Itally, Febuary 2006, pp. 176-181.

[2] J. A. Rosas-Flores, J. A. Flores-Campos, and L. G. Corona-Ramírez, "Optimal linearization of the dynamic behavior of an on/off actuated single pneumatic cylinder," in Proceedings of 2008 5th International Conference on Electrical Engineering, Computing Science and Automatic Control (CCE 2008), Mexico City, November 2008, pp. 380385.

[3] A. Girin, F. Plestan, X. Brun, and A. Glumineau, "High-order slidingmode controllers of an electropneumatic actuator: Application to an aeronautic benchmark," International Journal of Control, vol. 79, no. 2, pp. 119-131, 2006.

[4] K. Xing, J. Huang, Y. Wang, J. Wu, Q. Xu, and J. He, "Tracking control of pneumatic artificial muscle actuators based on sliding mode and non-linear disturbance observer," IET Control Theory and Applications, vol. 4, no. 10, pp. 2058-2070, 2010.

[5] T. Nguyen, J. Leavitt, F. Jabbari, and J. E. Bobrow, "Accurate slidingmode control of pneumatic systems using low-cost solenoid valves," IEEE/ASME Transactions on Mechatronics, vol. 12, no. 2, pp. 216219, 2007.

[6] X. Shen, J. Zhang, E. J. Barth, and M. Goldfarb, "Nonlinear modelbased control of pulse width modulated pneumatic servo systems," Journal of Dynamic Systems, Measurement, and Control, vol. 128, pp. 663-669, 2006.

[7] M. Q. Le, M. T. Pham, M. Tavakoli, and R. Moreau, "Sliding mode control of a pneumatic haptic teleoperation system with on/off solenoid valves," in Proceedings of IEEE International Conference on Robotics and Automation (ICRA), Shanghai, China, May 2011, pp. 874-879.

[8] V. Utkin, "Sliding mode control design principles and applications to electric drives," IEEE Transactions on Industrial Electronics, vol. 40, no. 1, pp. 23-36, 1993.

[9] M. Q. Le, M. T. Pham, R. Moreau, and T. Redarce, "Transparency of a pneumatic teleoperation system using on/off solenoid valves," in Proceedings of 19th IEEE International Symposium on Robot and Human Interactive Communication (RO-MAN), Viareggio, Itally, September 2010, pp. 15-20.

[10] P. Beater, Pneumatic Drives - System Design, Modelling and Control. Berlin: Springer, 2006.

[11] M. Q. Le, M. T. Pham, M. Tavakoli, and R. Moreau, "Development of a hybrid control for a pneumatic teleoperation system using on/off solenoid valves," in Proceedings of IEEE/RSJ International Conference on Intelligent Robots and Systems (IROS), Taipei, Taiwan, October 2010, pp. 5818 - 5823 . 